\title{
BioéthiqueOnline
}

\section{La lenteur ethnographique traduite pour les comités d'éthique de la recherche}

\section{Karine St-Denis}

Volume 6, 2017

URI : https://id.erudit.org/iderudit/1044620ar

DOI : https://doi.org/10.7202/1044620ar

Aller au sommaire du numéro

Éditeur(s)

BioéthiqueOnline

ISSN

1923-2799 (numérique)

Découvrir la revue

Citer cet article

St-Denis, K. (2017). La lenteur ethnographique traduite pour les comités d'éthique de la recherche. BioéthiqueOnline, 6.

https://doi.org/10.7202/1044620ar
Résumé de l'article

Cette étude du cas a comme objectif de traduire la lenteur ethnographique pour les membres des comités d'éthique de la recherche (CÉR). Nous montrerons que malgré les récents efforts de reconnaissance de la spécificité des recherches dites qualitatives en éthique de la recherche, une incompréhension demeure entre la rationalisation expérimentale déployée par plusieurs CÉR et la pratique de l'ethnographie une méthode de recherche par immersion lente et durable indissociable de relations humaines de confiance et de respect mutuel. 


\title{
La lenteur ethnographique traduite pour les comités d'éthique de la recherche
}

\author{
ÉTUDE DE CAS / CASE STUDY \\ Karine St-Denis ${ }^{1}$
}

Reçu/Received: 17 Jul $2017 \quad$ Publié/Published: 11 Dec 2017
Éditeurs/Editors: Patrick Gogognon \& Lise Lévesque

2017 K St-Denis, Creative Commons Attribution 4.0 International License

\section{Résumé}

Cette étude du cas a comme objectif de traduire la lenteur ethnographique pour les membres des comités d'éthique de la recherche (CÉR). Nous montrerons que malgré les récents efforts de reconnaissance de la spécificité des recherches dites qualitatives en éthique de la recherche, une incompréhension demeure entre la rationalisation expérimentale déployée par plusieurs CÉR et la pratique de l'ethnographie une méthode de recherche par immersion lente et durable indissociable de relations humaines de confiance et de respect mutuel.

\section{Mots clés}

éthique de la recherche, comité d'éthique de la recherche, CÉR, ethnographie, anthropologie, recherches qualitatives

\section{Summary}

This case study aims to explain ethnographic slowness for members of the Research Ethics Boards (REBs). I will show that despite recent efforts to recognize the specificity of so-called qualitative research in research ethics, a misunderstanding remains between the experimental rationalization deployed by several REBs and the practice of ethnography, a method of research involving slow and sustained immersion that is inseparable from human relations of trust and mutual respect.

\section{Keywords}

research ethics, research ethics board, REB, ethnography, anthropology, qualitative research

Affiliations des auteurs / Author Affiliations

${ }^{1}$ CIUSSS Nord-de-l'ile-de-Montréal, Montréal, Québec, Canada

\section{Correspondance / Correspondence}

Karine St-Denis, karine.st-denis@sympatico.ca

\section{Remerciements}

Nous remercions l'équipe éditoriale de BioéthiqueOnline ; leurs commentaires ont contribué à la qualité de cette étude de cas.

\section{Conflit d'intérêts}

Aucune déclaré

\section{Acknowledgements}

Thanks to the editorial team of BioéthiqueOnline; their comments contributed to the quality of this case study.

Conflicts of Interest

None to declare

\section{Introduction}

L'évaluation et l'approbation d'un projet de recherche par un Comité d'éthique de la recherche (CÉR) sont rarement posées comme un exercice de communication entre le chercheur et les membres du CÉR. Pourtant, il s'agit d'un réel exercice de traduction pour le chercheur qui doit réfléchir aux visées et méthodes habituelles de sa discipline pour les contenir dans des termes perceptibles et signifiants pour les membres des CÉR. Nous consacrerons cette étude de cas à cet exercice traductif. Nous montrerons que malgré les récents efforts de reconnaissance de la spécificité des recherches dites qualitatives ${ }^{1}$ en éthique de la recherche, une incompréhension demeure entre la rationalisation expérimentale déployée par plusieurs CÉR et la pratique de l'ethnographie, une méthode de

\footnotetext{
${ }^{1}$ Cette expression même de « recherche qualitative » est sujette à débats. Notamment pour Olivier de Sardan [1], la nature des données - soit qualitative et quantitative - n'est pas adéquate pour qualifier la méthode ethnographique puisque "L'enquête de terrain fait feu de tout bois. Son empirisme est résolument éclectique et s'appuie sur tous les modes de recueil de données accessibles » (p.71).
} 
recherche par immersion lente et durable qui est indissociable des relations humaines de confiance et de respect mutuel.

\section{La lenteur ethnographique: le chercheur engagé dans des relations humaines}

Débutons par quelques précisions sur cette immersion de longue durée auprès des populations étudiées. En anthropologie, la lenteur est posée et reconnue comme critère de validité des savoirs, et ce depuis les fondements de la discipline. ${ }^{2}$ Le conseil que Bernard Arcand reçut en début de recherche doctorale résonne aux oreilles des anthropologues: "Allez-y. Restez là 2 ans. Revenez puis vous nous raconterez quelque chose; parce qu'en 2 ans vous aurez à peu près le temps de commencer à peine à comprendre ce qui se passe » [3].

La lenteur imprègne toute la démarche ethnographique. Parvenir à interroger une réalité socioculturelle et à la comprendre demande écoute et silences, observations et patience. Elle demande à l'ethnologue de s'engager dans une relation humaine vraie et durable. ${ }^{3}$ Lorsqu'un CÉR le questionne sur ses activités de collecte de données et sur la nature de l'implication des participants, l'ethnologue voudra répondre : mais tout dépendra d'eux! Mais, il sait que cette réponse, la seule cohérente pour sa discipline, est peu audible par les membres des CÉR pour qui devis de recherche expérimentale, et par conséquent, outils de collecte formalisés et objectivation de la relation de recherche sont d'usages.

Pour résoudre cette difficulté, l'ethnologue a appris à construire des devis et des échéanciers de recherche répondant aux attentes. Comme il est d'usage en recherche expérimentale, son devis offrira des outils de collecte préétablis et un échéancier avec dates de tombées pour sa collecte de données, son analyse et pour la diffusion des résultats, et ce, dans un format plausible pour les CÉR. Mais, une fois sur le terrain, sa réalité et sa temporalité seront souvent toute autre.

L'ethnologue n'aura alors rien dit des enjeux engendrés par la lenteur de sa discipline. II n'aura rien dit de son engagement bien plus que scientifique auprès de ses participants de recherche. II n'aura rien dit de son engagement humain empreint de respect et de confiance mutuels. II n'aura rien dit de ses amitiés. ${ }^{4}$ Pour plus d'un, se taire évitera les questionnements des profanes et l'embourbement dans des autorisations de déviations au protocole et au processus de consentement. Pour d'autres, se taire dit toute la difficulté de traduire cette lenteur avec les termes d'usage en sciences expérimentales et en éthique de la recherche [5].

Dans les prochaines sections, nous tentons simplement de dire en quoi la lenteur intrinsèque de la méthode ethnographique engage l'ethnologue dans des pratiques particulières de respect et de bienêtre des participants. Pour ce faire, nous utilisons un récit d'expérience ethnographique, celui du sociologue français Romain Pudal [6]. ${ }^{5}$ Ce récit d'expérience nous servira, premièrement, à montrer la nécessité méthodologique de cette lenteur. Dans un second temps, ce récit nous permettra de nommer les choix et pratiques éthiques des ethnologues.

\footnotetext{
${ }^{2}$ Voir notamment Evans-Pritchard (1902-1973), auteur fondateur de la méthode ethnographique, pour qui : « It is almost impossible for a person who knows what he is looking for, and how to look for it, to be mistaken about the facts if he spends two years among a small and culturally homogeneous people doing nothing else but studying their way of life. " [2, p.83]

${ }^{3}$ Plusieurs débats ont lieu en anthropologie sur la nature de cette implication du chercheur. Celle-ci peut aller de l'acceptation de la subjectivité du chercheur à une imprégnation totale qualifiée de " going native » [1].

${ }^{4}$ Cette amitié et ses conséquences sont rarement affirmées dans les ethnographies. On la retrouve plus aisément relayée dans les préfaces et les postfaces. Un ouvrage récent d'un anthropologue québécois, $P$. Maranda, se distingue ici [4].

${ }^{5}$ Nous avons délibérément préféré un récit d'expérience publié par un autre chercheur plutôt que de rendre compte d'une expérience personnelle. Nous souhaitions ainsi montrer que ces expériences sont partagées par plus d'un chercheur. Avouons également que, comme nos travaux ethnologiques sont toujours en cours, publier nos expériences demeure délicat en matière de confidentialité.
} 


\section{La lenteur ethnographique : une nécessité méthodologique}

Dans son ouvrage Retour de flammes. Les pompiers, des héros fatigués ? [6], le sociologue et pompier volontaire Romain Pudal offre une auto-ethnographie de ses confrères de casernes. Ses récits de formations et d'opérations y côtoient une analyse critique des enjeux de recrutements et des conséquences professionnelles de la transformation du métier et des conditions sociologiques de sa réalisation. L'auteur y offre aussi un récit détaillé d'une de ses entrevues de recherche à titre de chargé d'études au Service départemental d'incendie et de secours (SDIS) ${ }^{6}$; c'est sur ce récit que nous porterons notre attention.

Lors de ses heures de gardes en caserne, Pudal réalise des entrevues avec ses confrères pompiers. Ces entrevues vont bon train, mais l'une d'elles le questionne :

Au début, Thierry répond à mes questions un peu mécaniquement, presque dans la vague, jouant avec son bip, sans jamais en dire beaucoup. Nous convenons assez rapidement que l'entretien doit se terminer, qu'il se fait tard (ce qui n'a aucun sens en réalité quand on est de garde). J'ai un peu de mal à me l'avouer, mais je suis déçu et m'en veux de n'avoir pas su m'y prendre [6, p.76].

Plusieurs semaines plus tard, Pudal en fin de garde, se croyant seul en caserne, décide de se rendre à la salle de sport. C'est alors que Thierry vient le rejoindre pour un long entretien.

Nous nous retrouvons seuls dans une caserne désertée, assis sur des appareils de musculation; je suis en short et t-shirt, sans dictaphone évidemment. On peut dire : c'est bien Thierry qui aura décidé des conditions de passation de l'entretien [6, p.77].

Ce récit n'est pas une expérience rarissime. Bien au contraire, le terrain ethnographique est fait de ses instants où la qualité de la relation humaine surpasse la relation chercheur-participant [1]. Le terrain est fait de ces instants où la confiance mutuelle s'exprime au-delà des devis de recherche, des standards méthodologiques et des certifications éthiques. Ces instants ne peuvent pas être vécus sans la lenteur ethnographique, sans cette durée de la présence mutuelle.

\section{La lenteur ethnographique: des pratiques spécifiques en matière de respect et de bien-être des participants}

Faire sienne cette lenteur ethnographique s'accompagne de choix éthiques. Elle engage subjectivement le chercheur dans une série de préférences et de pratiques notamment en matière de respect et de bien-être du participant. Examinons de nouveau le récit d'entrevue de Pudal non plus en termes méthodologiques, mais plutôt avec le vocabulaire éthique d'usage en matière de respect et de bien-être du participant.

Débutons par une précision terminologique. Lors de l'évaluation et de l'approbation d'un projet de recherche par un CÉR, le respect des participants s'évalue principalement par la capacité du chercheur à respecter l'autonomie décisionnelle des participants. Le mécanisme du consentement libre éclairé et continu est privilégié pour certifier l'autonomie décisionnelle [7]. Quant à lui, le bienêtre, sans y être réduit, s'évalue principalement en termes de risques et bénéfices physiques, mentaux, spirituels, matériels, économiques et sociaux [7].

\footnotetext{
${ }^{6}$ Expression d'usage pour désigner les services de sécurité incendie et de premières réponses médicales en France.
} 
Reprenons le début du récit d'entrevue. Le participant, Thierry, a vraisemblablement offert son consentement avant de répondre aux questions d'entrevue. Par contre, il n'offre que des réponses mécaniques. Rapidement, l'entrevue est close pour un motif, qui comme le mentionne Pudal, n'a aucun sens lors d'une garde en caserne [6, p.76]. En n'appliquant que les normes d'éthique de la recherche, la situation n'est pas problématique: le consentement a été obtenu et devant des observations confirmant l'inconfort, le mal-être, du participant, l'entrevue est cessée.

Pourtant, Pudal sait qu'il a failli et il en est déçu. II sait que sa relation avec ses participants, ici avec Thierry, est une relation humaine qui, comme toutes relations humaines, est sujette à être chamboulée par une telle situation inconfortable. Pudal sait que, hors de la salle d'entrevue, Thierry est son collègue. Ils partagent le même quotidien en caserne : ils répondront aux appels, vérifieront les équipements, prendront leurs repas et se détendront ensemble. Par conséquent, il sait que, hors de la relation chercheur-participant, Thierry et Romain vivront et interagiront ensemble, dans le même environnement physique et social.

Ce qu'il advient quelques semaines plus tard dans la salle de sport de la caserne illustre que les principes de respect et de protection du bien-être s'incarnent bien au-delà de l'entretien formalisé. La lenteur et la durée de la présence de l'ethnologue le conduiront à ces moments où l'application des principes cède sa place à l'incarnation des principes. En ethnologie, la durée des échanges entre chercheur et participants permet aux relations interpersonnelles, voire amicales, de dépasser la formalisation expérimentale de la recherche.

Dans cette salle de sport, ce n'est plus le chercheur qui questionne un participant. II y a eu renversement des rôles: "C'est bien Thierry qui aura décidé des conditions de passation de l'entretien » [6, p.77]. Le chercheur - en short et sans son dictaphone - sait qu'il n'y a alors plus devis, méthodes, ni mécanismes de consentement aptes à dire la richesse de ce moment où la lenteur et la durée ethnographique parviennent à ce niveau de confiance et de respect. Dans cette salle de sport, il n'y a que Romain, Thierry et la reconnaissance mutuelle de leur interrelation.

Devant cette incarnation des principes éthiques de respect des participants et de la protection de leur bien-être, l'ethnologue ne saisira pas en quoi une consignation de consentement tacite ou une demande de déviation au protocole sont porteurs de sens.

\section{Conclusion : dire la lenteur ethnographique}

Héritière des sciences expérimentales, l'éthique de la recherche n'en est qu'à ses débuts en matière d'énonciation de la spécificité de la recherche qualitative. ${ }^{7}$ Ses cadres normatifs et ses pratiques, bien que signifiantes pour plusieurs chercheurs, sied mal à l'ethnologue. Comme le récit d'entrevue de Romain Pudal nous a permis de le constater, la lenteur ethnographique engage l'ethnologue dans des relations humaines complexes où s'entrelacent confiance, respect et amitié. Cette complexité se perd lors de sa traduction en termes de mécanismes de consentement et d'évaluation éthique de la recherche.

Devant un CÉR, l'ethnologue a une position inconfortable : mal-dire ou se taire. D'autant plus que pour lui la lenteur ethnographique est un lieu commun puisque, au-delà de la méthode, elle imprègne son éthos. II ne peut, à chaque fois, que s'étonner lorsque parler de cette lenteur résonne comme l'écho d'une salle de sport vide. Pour parvenir à s'entendre mutuellement, membres de CÉR et ethnologues doivent parvenir à parler le même langage. Dire la spécificité des recherches

\footnotetext{
${ }^{7}$ Voir notamment le chapitre 13 de l'Énoncé de politique de la recherche des trois Conseils [7] dont le vocabulaire et le contenu relèvent bien plus d'une introduction à la recherche qualitative qu'à un examen spécifique des enjeux éthiques inhérents à ces méthodes. Notons ici l'hétérogénéité des pratiques des CÉR. Certains CÉR et subventionnaires québécois majeurs demeurent réticents à la reconnaissance de la variabilité des mécanismes de consentement (écrit, oral, tacite), variabilité pourtant posée dans la version 2014 de l'EPTC.
} 
ethnographiques nécessite un dialogue entre deux traditions de recherche aux rationalités distinctes. Pour s'entendre, il faudra porter oreilles non seulement à la nature qualitative des données, mais bien à l'éthos des chercheurs qualifiés de qualitatifs.

\section{Questions}

Le récit d'entrevue présenté ici peut être analysé sous d'autres angles. Voici quelques questions pour en compléter la réflexion.

1. Quels motifs personnels et sociaux pourraient amener un participant à consentir à une entrevue de recherche ethnographique bien qu'il soit inconfortable avec les démarches de recherche?

2. Octroyer un temps de réflexion supplémentaire avant de consentir à l'entrevue résoudrait-il cet inconfort?

3. Quels défis la méthode ethnographique impose-t-elle au processus de consentement écrit?

4. Quelles adaptations du processus de consentement permettrait la flexibilité requise en ethnologie? Comment justifier ces adaptations à un comité d'éthique de la recherche CÉR?

5. Comment un chercheur devrait-il réagir face à cet inconfort d'un participant? Cesser l'entrevue est-il la seule solution?

\section{Références}

1. Olivier de Sardan J.-P. La rigueur du qualitatif, Louvain-la-Neuve, Academia-Bruylant, 2008.

2. Evans-Pritchard E.E. Social Anthropology, London, University Press Glascow, 1951.

3. Arcand B., Indicatif présent, Radio Canada, 12 janvier 2001.

4. Maranda, P. Ces Lau que j'ai tant aimé, Montréal, Nouvelle Optique, 2010.

5. L'American Anthropological Association. American Anthropological Association Statement on Ethnography and Institutional Review Boards, 2004.

6. Pudal R. Retour de flammes. Les pompiers, des héros fatigués? Éditions La Découverte, 2016.

7. Conseil de recherches en sciences humaines du Canada, Conseil de recherches en sciences naturelles et en génie du Canada, Instituts de recherche en santé du Canada. Énoncé de politique des trois Conseils : Éthique de la recherche avec des êtres humains, décembre 2014. 